\title{
Geometrical haptic illusions revisited: Haptic illusions compared with visual illusions
}

\author{
KOTARO SUZUKI and RYOKO ARASHIDA \\ Niigata University, Niigata, Japan
}

\begin{abstract}
Révész (1934) reported that haptic illusions were observed in almost all of the geometrical optical illusion figures. The present study reexamined seven geometrical illusions in both haptic and visual modes. In the Müller-Lyer, Ponzo, and vertical-horizontal figures, haptic illusions equivalent to the visual illusions were observed. In the Oppel-Kundt figure, a haptic illusion similar to the visual one was obtained. In the haptic Delboeuf stimuli, the size illusion of the outer circle occurred, whereas that of the inner circle did not. No haptic illusion was obtained in the Poggendorff figure. In the Zöllner figure, a haptic illusion directionally opposite to the visual one was obtained. These results show that haptic illusions do not occur in all of the geometrical illusion figures. They also suggest that haptic illusions are not necessarily mediated by visualization and that haptic processing of the figures often occurs in a manner different from vision.
\end{abstract}

More than 50 years ago, Révész (1934) reported that geometrical illusions were obtained in a haptic mode. He used 29 geometrical illusion figures and asked blindfolded and blind subjects to inspect the stimuli haptically. He obtained haptic illusions similar to the visual ones in almost all of the figures.

Since then, many researchers have examined his findings in several illusions by using blindfolded, congenitally blind, and late-blind subjects. For haptic stimuli, they used raised figures, braille dot figures, embossed figures, and so on. Among the geometrical haptic illusions, the Müller-Lyer illusion is frequently studied. This illusion was confirmed to exist in a haptic mode by several researchers (Bean, 1938; Békésy, 1967; Frisby \& Davies, 1971; Fry, 1975; Hatwell, 1960; Over, 1966; Patterson \& Deffenbacher, 1972; Révész, 1934; Rudel \& Teuber, 1963; Tsai, 1967). Also, the haptic illusion was shown to occur in the vertical-horizontal figures (Bean, 1938; Day \& Wong, 1971; Fry, 1975; Hatwell, 1960; Over, 1966; Révész, 1934; Wong, 1977). The Ponzo illusion was reported to be observed in the haptic mode (Bean, 1938; Révész, 1934), as was also the Zöllner illusion (Bean, 1938). The Oppel-Kundt figures also induced a haptic illusion (Békésy, 1967; Révész, 1934).

Not all of the geometrical optical illusions, however, could be obtained in a haptic mode. In the Poggendorff figures, some studies reported a haptic illusion (Bean, 1938; Pasnak \& Ahr, 1970; Révész, 1934), but Wenderoth and Alais (1990) could not obtain any haptic illusion by reexamining the haptic Poggendorff illusion directionally opposite to the visual one reported by Lucca,

We are grateful to Hitoshi Honda and Naofumi Fujita for their helpful comments on an earlier version of the manuscript. Correspondence should be addressed to Kotaro Suzuki, Department of Psychology, Faculty of Humanities, Niigata University, Ikarashi, Niigata 950-21, Japan.
Dellantonio, and Riggio (1986). Thus, whether or not a haptic Poggendorff illusion can be induced is questionable. Also, Hatwell (1960) reported no haptic illusion in the Delboeuf figure, although she dealt only with the size illusion of the inner circle. Fry (1975) obtained a haptic Delboeuf illusion directionally opposite to the visual one; he used concentric squares as stimuli but did not report whether he dealt with the size illusion of the inner or the outer square. Thus, the haptic Delboeuf illusion is also doubtful, further indicating that not all of the geometrical optical illusions can be observed in the haptic mode. The present study examines which geometrical illusion can be induced in the haptic mode and which cannot be.

Except for Révész's (1934) study, the above-mentioned studies examined only one or, at best, a few illusions. The present study examines seven illusions: Müller-Lyer, Oppel-Kundt, Ponzo, Poggendorff, vertical-horizontal, Zöllner, and Delboeuf illusions. However, Révész did not examine the Zöllner and Delboeuf illusions. We chose these seven illusions because they are well-examined in vision and are typical among the similar distortion types of illusions. In the taxonomy for illusory distortions proposed by Coren, Girgus, Erlichman, and Hakstian (1976), the Müller-Lyer, Oppel-Kundt, and vertical-horizontal illusions were classified as the illusions of extent, and the Ponzo, Zöllner, and Delboeuf illusions were classified as the illusions of shape or direction. Some of the illusions of shape or direction might not occur in a haptic mode, since either no illusion or a directionally opposite illusion to the visual one was reported in the haptic Delboeuf stimuli (Fry, 1975; Hatwell, 1960). Also, the Poggendorff illusion, which was classified as neither type of illusion in Coren et al. (1976), might not be obtained in a haptic mode, since Wenderoth and Alais (1990) reported negative results in the haptic Poggendorff illusion.

In the present study, we also compare the results obtained in a haptic mode with those obtained in a visual 
mode. By comparing the haptic and visual illusions quantitatively, we can consider an equivalence or difference between them. To obtain exact quantitative measures, we used the up-and-down method as our method of measurement, and the comparison stimuli varied by small steps.

In the 1960s, the "inappropriate constancy scaling" hypothesis for geometrical optical illusions proposed by Gregory (1963) became prevalent as an explanation for visual illusions. If haptic illusions are induced by the geometrical illusion figures, however, they should be an obstacle to this hypothesis, since haptic illusion figures do not convey any information about depth (Over, 1967). However, some researchers claimed that the illusions obtained in a haptic mode were mediated by visualization (e.g., Appelle \& Gravetter, 1985; Frisby \& Davies, 1971). Therefore, Gregory's hypothesis can also be applied in the haptic illusions. There is, however, a report that the haptic Müller-Lyer illusion was obtained in the congenitally blind, who could not use visualization (Bean, 1938; Patterson \& Deffenbacher, 1972), which suggests that haptic illusions are not mediated by visualization. The present study also examines this issue. That is, if some of the geometrical illusions are obtained in the haptic mode whereas others are not, visualization should not necessarily be involved in haptic illusions.

\section{METHOD}

\section{Subjects}

For each geometrical illusion, 16 sighted undergraduate students served as subjects. Not all of the subjects were used for all illusions, but about half of the subjects participated in common across different illusions.

\section{Stimuli}

The geometrical illusion figures used in the experiment are shown in Figure 1. Haptic stimulus figures were raised on sheets of paper for braille use (available from the Japan Braille Library). Visual stimulus figures were drawn with black ink on sheets of white drawing paper. The width of the raised or drawn lines was $1 \mathrm{~mm}$. The size of the sheets was $18.2 \times 25.7 \mathrm{~cm}$ in the Müller-Lyer, OppelKundt, and Delboeuf figures, and $25.7 \times 18.2 \mathrm{~cm}$ in the Ponzo, Poggendorff, vertical-horizontal, and Zöllner figures.

To take into account the possible anisotropy of space, we generally used two types of stimuli, except with the vertical-horizontal and Zöllner figures. In one type of stimulus sheet, the standard figure (or line or circle) was on the right and the comparison figure on the left (called the left condition here). In the other type, the positions of the standard and comparison figures were exchanged (called the right condition here). Both types of stimulus sheets were presented to each subject.

Each illusion figure is as follows:

Müller-Lyer figure. Ingoing and outgoing figures were arranged side by side (Figure 1a). Standard and comparison stimuli were the outgoing and ingoing figures, respectively. They were $20 \mathrm{~mm}$ apart from each other. The shaft (central line) of the outgoing figure was $30 \mathrm{~mm}$ long. The length of each fin was $10 \mathrm{~mm}$, and the angle between the fins was $90^{\circ}$. In the comparison figure, the length of the shaft varied from $15-45 \mathrm{~mm}$ by a step of $1 \mathrm{~mm}$, resulting in 31 different stimulus sheets for each left-right condition.

Oppel-Kundt figure. The standard stimulus was a filled extent $40 \mathrm{~mm}$ wide, which was divided by lines $10 \mathrm{~mm}$ high at intervals of $5 \mathrm{~mm}$ (Figure $1 \mathrm{~b}$ ). The comparison stimulus was an unfilled extent. In the comparison stimuli, the unfilled extent was varied from

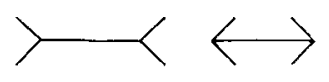

a
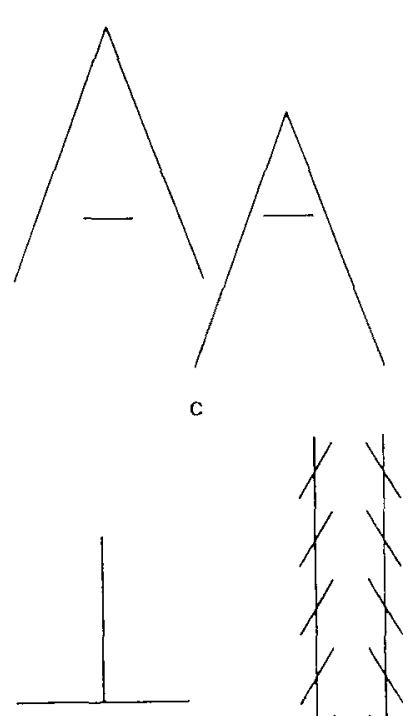

e
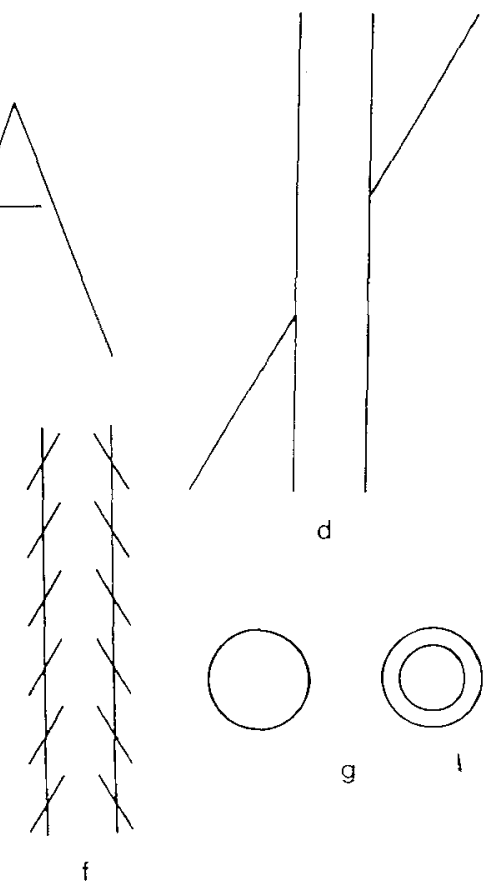

g

Figure 1. Geometrical illusion figures that were used in the experiment. Patterns in the left condition, in which the comparison figure was arranged on the left, are shown as examples. The figures are (a) Müller-Lyer, (b) Oppel-Kundt, (c) Ponzo, (d) Poggendorff, (e) vertical-horizontal, (f) Zöllner, and (g) Delboeuf.

25-55 mm by a step of $1 \mathrm{~mm}$, resulting in 31 stimulus sheets for each left-right condition.

Ponzo figure. Two converging angles of $40^{\circ}$ were arranged obliquely side by side (Figure 1c). Converging lines of the angles were $105 \mathrm{~mm}$ long. The standard stimulus was a horizontal line $20 \mathrm{~mm}$ long, $40 \mathrm{~mm}$ apart from the converging point within the angle. The comparison stimulus was a horizontal line, $75 \mathrm{~mm}$ apart from the converging point. The length of the comparison line was varied from $10-30 \mathrm{~mm}$ by a step of $1 \mathrm{~mm}$, resulting in 21 stimulus sheets for each left-right condition.

Poggendorff figure. Two parallel vertical lines, $24 \mathrm{~mm}$ apart from each other and $160 \mathrm{~mm}$ long, were intersected by oblique lines (Figure 1d). The angle of intersection was $30^{\circ}$. The upper oblique line was used as the standard line, and the lower oblique line as the comparison line. The position of the lower oblique comparison line was varied along the vertical line from $30 \mathrm{~mm}$ upper to $30 \mathrm{~mm}$ lower, by a step of $2 \mathrm{~mm}$, from the position to be passed by the upper oblique line. Thus, 31 stimulus sheets were made for each left-right condition. In all of the figures, the lower oblique line ended at the level of the lower end of the vertical lines.

Vertical-horizontal figure. The stimulus figure is shown in Figure le. The standard stimulus was a 50-mm-long horizontal line. The comparison stimulus was a vertical line that joined the horizontal line at its center. The length of the vertical comparison line was varied from 30-70 mm by a step of $1 \mathrm{~mm}$, resulting in 41 stimulus sheets. In the vertical-horizontal figure, the left-right position was not relevant. 
Zollner figure. Two types of stimulus figures were used: two vertical lines intersected by converging-downward oblique lines (called here downward-oblique type) and two vertical lines intersected by converging-upward oblique lines (upward-oblique type). The upward-oblique type is shown in Figure 1f. Two vertical lines of $120 \mathrm{~mm}$ in height were $20 \mathrm{~mm}$ apart at the center points. Each vertical line was intersected by six oblique lines $20 \mathrm{~mm}$ long. The angle of intersection was $30^{\circ}$. In the stimulus series, the orientation of the two vertical lines was co-varied by a step of $1 \mathrm{~mm}$. When the bottom width between the two lines widened $1 \mathrm{~mm}$, their top width narrowed $1 \mathrm{~mm}$. Thus, 21 stimulus sheets were made for each type of figure.

Delboeuf figure. The stimulus figure is shown in Figure $1 \mathrm{~g}$. We examined the Delboeuf illusion relative to two measures: perceived sizes of the inner and outer circles. The standard figure was the inner or outer circle of the double concentric circles. The diameters of the inner and outer circles were 20 and $30 \mathrm{~mm}$, respectively. The diameter of the comparison circle was varied from 14-36 mm by a step of $1 \mathrm{~mm}$. Thus, 23 stimulus sheets were made for each left-right condition.

\section{Procedure}

In the haptic condition, a blindfolded subject was asked to trace the raised stimulus sheet on a table with the fingers, mainly with the index finger, of his or her right hand. The subject was instructed to inspect the entire figure haptically in each trial. In the visual condition, the figures were presented on a blackboard, $45 \times 40 \mathrm{~cm}$, at the subject's eye-level. The viewing distance was $50 \mathrm{~cm}$, which corresponded approximately to the distance from the subject's eyes to the stimulus sheet in the haptic condition.

The subject was asked whether he or she perceived the comparison figures as longer or shorter (larger or smaller, wider or narrower, or higher or lower) than the standard figures. In the Zöllner figure, the subject was asked whether he or she perceived the two vertical lines as converging upward or downward. In the Delboeuf figure, the subject made size judgments for both the inner and outer circles of the double concentric circles.

The up-and-down method was used: one ascending and one descending series were executed for each condition. In each series, the trials were ended when the subject's judgment reversed in direction six times. Points of subjective equality (PSEs) were calculated from the 12 values obtained in the ascending and descending series.

The orders of the conditions (visual/haptic and left/right) were counterbalanced among the subjects. The presentation orders of ascending and descending series were also counterbalanced.

\section{RESULTS}

\section{Müller-Lyer Illusion}

Table 1 shows mean error scores for each condition. The illusion occurred in both the haptic and visual conditions: The shaft of the outgoing figure appeared approxi-

Table 1

Mean Error Scores (in Millimeters) in the Müller-Lyer Figure

\begin{tabular}{|c|c|c|c|c|c|c|}
\hline & \multicolumn{6}{|c|}{ Condition } \\
\hline & \multicolumn{3}{|c|}{ Haptic } & \multicolumn{3}{|c|}{ Visual } \\
\hline & $M$ & $S D$ & Ratio & $M$ & $S D$ & Ratic \\
\hline Left & 8.3 & 2.88 & 1.28 & 8.3 & 1.70 & 1.28 \\
\hline Right & 9.6 & 1.99 & 1.32 & 8.6 & 2.15 & 1.29 \\
\hline$M$ & 9.0 & 2.14 & 1.30 & 8.5 & 1.87 & 1.28 \\
\hline
\end{tabular}

Note-The length of the standard shaft was $30 \mathrm{~mm}$. Left and Right refer to the comparison stimulus on the left and on the right. Ratio is the magnitude of the illusion, that is, the ratio of the length of the comparison shaft to that of the standard shafi.
Table 2

Mean Error Scores (in Millimeters) in the Oppel-Kundt Figure Condition

\begin{tabular}{|c|c|c|c|c|c|c|}
\hline & \multicolumn{3}{|c|}{ Haptic } & \multicolumn{3}{|c|}{ Visua! } \\
\hline & $M$ & $S D$ & Ratio & $M$ & $S D$ & Ratio \\
\hline Left & 2.5 & 4.22 & 1.06 & 6.3 & 1.89 & 1.16 \\
\hline Right & 2.2 & 4.32 & 1.05 & 5.7 & 2.71 & 1.14 \\
\hline$M$ & 2.3 & 3.93 & 1.06 & 6.0 & 2.06 & 1.15 \\
\hline
\end{tabular}

Note-The width of the standard filled extent was $40 \mathrm{~mm}$. Left and Right refer to the comparison stimulus on the left and on the right. Ratio is the magnitude of the illusion, that is, the ratio of the width of the comparison unfilled extent to that of the standard filled extent.

mately 1.3 times longer than that of the ingoing figure. To show whether the obtained error scores significantly differed from zero, $t$ tests for the single means were performed. The one-tailed test was used for the visual condition, in which a directional prediction of the illusion had been made. The two-tailed test was used for the haptic condition, in which a nondirectional prediction had been made. The illusions significantly occurred $[t(15)=16.20$, $p<.0001$, two-tailed for the haptic condition, and $t(15)=17.50, p<.0001$, one-tailed for the visual condition]. There was no difference in error scores between the haptic and visual conditions $[F(1,15)=1.22$, n.s.] There was a small significant difference in error scores between left-right position conditions $[F(1,15)=5.54$, $p<.05]$, but no interaction was found between the left-right conditions and the haptic-visual conditions $[F(1,15)=1.81$, n.s. $]$.

The results coincide with those obtained in other studies (Bean, 1938; Békésy, 1967; Fry, 1975; Hatwell, 1960; Over, 1966; Patterson \& Deffenbacher, 1972; Révész, 1934; Rudel \& Teuber, 1963; Tsai, 1967). The magnitude of the haptic Müller-Lyer illusion was not different from the visual one, suggesting an equivalence between them.

\section{Oppel-Kundt Illusion}

The results are shown in Table 2 . In both the haptic and visual conditions, the illusion was obtained: The filled extent appeared wider than the unfilled one (1.06 times in the haptic condition and 1.15 times in the visual condition). The visual illusion was significant $[t(15)=11.38$, $p<.0001$, one-tailed]; the haptic illusion was also significant $[t(15)=2.29, p<.05$, two-tailed]. The difference in error scores between the haptic and visual conditions was not significant $[F(1,15)=3.43, .10>p>$ $.05]$. The left-right position did not affect the illusion $[F(1,15)=1.17$, n.s. $]$. Therefore, we can say that the magnitude of the haptic Oppel-Kundt illusion was similar to that of the visual one.

The haptic Oppel-Kundt illusion was examined by Békésy (1967) and Révész (1934). Békésy obtained the illusion, but Révész reported that there were large individual differences in the magnitude of the illusion. We obtained the haptic illusion, but its variability was larger than that for the visual one, and a few subjects underestimated the filled extent. These results agree with 
Table 3

Mean Error Scores (in Millimeters) in the Ponzo Figure

\begin{tabular}{lccccccc}
\hline & \multicolumn{5}{c}{ Condition } \\
\cline { 2 - 6 } & \multicolumn{3}{c}{ Haptic } & & \multicolumn{3}{c}{ Visual } \\
\cline { 2 - 7 } & $M$ & $S D$ & Ratio & & $M$ & $S D$ & Ratio \\
\hline Left & 2.7 & 1.80 & 1.14 & 1.4 & .60 & 1.07 \\
Right & 1.9 & 1.70 & 1.10 & 1.4 & .98 & 1.07 \\
$M$ & 2.3 & 1.50 & 1.12 & 1.4 & .70 & 1.07 \\
\hline
\end{tabular}

Note-The length of the standard line was $20 \mathrm{~mm}$. Left and Right refer to the comparison stimulus on the left and on the right. Ratio is the magnitude of the illusion, that is, the ratio of the length of the comparison line to that of the standard line.

Table 4

Error Scores (in Millimeters) in the Poggendorff Figure

\begin{tabular}{|c|c|c|c|c|c|c|}
\hline \multirow[b]{3}{*}{ Subject } & \multicolumn{6}{|c|}{ Condition } \\
\hline & \multicolumn{3}{|c|}{ Haptic } & \multicolumn{3}{|c|}{ Visual } \\
\hline & Left & Right & $M$ & Left & Right & $M$ \\
\hline 1 & 18.0 & 13.7 & 15.8 & -11.5 & -9.8 & -10.7 \\
\hline 2 & 1.8 & 3.5 & 2.7 & -5.4 & -6.8 & -6.1 \\
\hline 3 & 1.2 & -2.8 & -.8 & -14.5 & -9.7 & -12.1 \\
\hline 4 & -11.7 & 3.3 & -4.2 & -3.8 & -7.7 & -5.8 \\
\hline 5 & -10.8 & -8.0 & -9.4 & -11.8 & -11.7 & -11.8 \\
\hline 6 & 12.6 & 3.3 & 8.0 & -6.5 & -9.2 & -7.8 \\
\hline 7 & -11.5 & -4.8 & -8.2 & -15.3 & -14.3 & -14.8 \\
\hline 8 & -10.8 & -2.0 & -6.4 & -10.8 & -15.3 & -13.1 \\
\hline 9 & 2.5 & .0 & 1.3 & -9.5 & -7.3 & -8.4 \\
\hline 10 & -12.4 & -9.0 & -10.7 & -10.3 & -13.2 & -11.8 \\
\hline 11 & 1.3 & -7.7 & -3.2 & -14.3 & -15.0 & -14.7 \\
\hline 12 & -7.0 & -5.3 & -6.2 & -7.5 & -13.0 & -10.3 \\
\hline 13 & -7.3 & -9.5 & -8.4 & -8.8 & -13.3 & -11.1 \\
\hline 14 & -5.7 & 17.7 & 6.0 & -10.0 & -7.5 & -8.8 \\
\hline 15 & -13.0 & 7.3 & -2.8 & -14.0 & -13.5 & -13.8 \\
\hline 16 & 14.0 & -9.0 & 2.5 & -5.0 & -10.5 & -7.8 \\
\hline $\mathbf{M}$ & -2.4 & -.6 & -1.5 & -10.0 & -11.1 & -10.6 \\
\hline$S D$ & 10.19 & 8.25 & 7.24 & 3.60 & 2.82 & 2.78 \\
\hline
\end{tabular}

Note-Positive values indicate the upward displacement of the lower obliques from the objectively accurate position, and negative values indicate the downward displacement. Left and Right refer to the comparison stimulus on the left and on the right.

Révész's (1934). Some studies on the tactual space (that is, space perceived by the passive touch) reported that the filled space tends to be perceived as smaller than the unfilled space (Craig, 1931; Parrish, 1895). This discrepancy in results might be due to the manner of touching, that is, active or passive touching. In active touching, we can say that the haptic Oppel-Kundt illusion is similar to the visual one.

\section{Ponzo Illusion}

The results are shown in Table 3. The illusion occurred in both the haptic and visual conditions: The horizontal line nearer to the converging point appeared 1.07 to 1.10 times longer than the line farther from the converging point. The $t$ tests for the single means showed that a significant illusion occurred $[t(15)=6.03, p<.0005$, twotailed for the haptic condition, and $t(15)=8.00, p<$ .0005 , one-tailed for the visual condition]. There was no difference in error scores between the haptic and visual conditions $[F(1,15)=1.34$, n.s. $]$. The difference in error scores between the left-right position conditions was not significant $[F(1,15)=4.37, .10>p>.05]$, and no interaction was found between the left-right conditions and the haptic-visual conditions $[F(1,15)=2.93$, n.s. $]$.

The results show that the Ponzo illusion occurs in the haptic mode, which agrees with Bean's (1938) and Révész's (1934) results. The magnitude of the haptic illusion was the same as that of the visual one. Therefore, we can say that the haptic Ponzo illusion is equivalent to the visual one.

\section{Poggendorff Illusion}

Table 4 shows the error scores for each subject for each condition. The illusion was obtained in the visual condition $[t(15)=13.72, p<.0001$, one-tailed $]$. In the haptic mode, however, there was a large variability of error scores between the left and right conditions as well as among the subjects. Statistically, no illusion occurred $[t(15)=.81$, n.s., two-tailed $]$. The difference in error scores between the haptic and visual conditions was highly significant $[F(1,15)=30.61, p<.0001]$. The left-right position did not affect the error scores $[F(1,15)=.41$, n.s.].

The results contrast with Bean's (1938), Pasnak and Ahr's (1970), and Révész's (1934) results but agree with Wenderoth and Alais' (1990) results. Wenderoth and Alais reexamined Lucca et al.'s (1986) finding that the haptic Poggendorff illusion was directionally opposite to the visual one. They concluded that this finding was probably an artifact because of the use of raised stimuli; no haptic Poggendorff illusion occurred in grooved stimuli. In our experiment, however, no Poggendorff illusion was obtained even in raised stimuli.

\section{Vertical-Horizontal Illusion}

Table 5 shows the obtained mean error scores. The illusion was obtained in both haptic and visual conditions: The vertical line was perceived as more than 1.2 times longer than the horizontal line $[t(15)=12.99, p<.0001$, one-tailed for the visual condition, and $t(15)=15.93$, $p<.0001$, two-tailed for the haptic condition]. There was no difference in error scores between these conditions $[F(1,15)=.25$, n.s.].

The results coincide with those obtained by other researchers (Bean, 1938; Day \& Wong, 1971; Fry, 1975; Hatwell, 1960; Over, 1966; Révész, 1934; Wong, 1977). It was shown that the haptic vertical-horizontal illusion is determined by the difference between radial and tangential movements of hand and arm (Cheng, 1968; Day

Table 5

Mean Error Scores (in Millimeters) in the Vertical-Horizontal Figure

\begin{tabular}{lll}
\hline & \multicolumn{2}{c}{ Condition } \\
\cline { 2 - 3 } & Haptic & Visual \\
\hline$M$ & 11.3 & 11.8 \\
$S D$ & 2.74 & 3.52 \\
Ratio & 1.23 & 1.24 \\
\hline
\end{tabular}

Note-The length of the standard horizontal line was $50 \mathrm{~mm}$. Ratio is the magnitude of the illustion, that is, the ratio of the length of the comparison line to that of the standard line. 
\& Wong, 1971; Wong, 1977). In our experiment, the subjects inspected the haptic stimuli by moving their hands radially and tangentially. Therefore, the haptic illusion obtained here could be a radial-tangential one, not really a vertical-horizontal one.

\section{Zöllner Illusion}

Table 6 shows the mean error scores for each type of figure. In the visual condition, the expected distortion of line orientation occurred in both types of figures $[t(15)=$ $6.22, p<.0005$, one-tailed for the downward-oblique type, and $t(15)=6.51, p<.0005$, one-tailed for the upward-oblique type]. In the haptic condition, the illusion occurred $[t(15)=2.94, p=.01$, two-tailed for the downward-oblique type, and $t(15)=7.81, p<.001$, two-tailed for the upward-oblique type], but the vertical lines were distorted in the opposite direction of the visual Zöllner illusion. The visual and haptic illusions clearly differed from each other in both types of figure $[F(1,15)=38.58, p<.0001$, for the downward-oblique type and $F(1,15)=80.63, p<.0001$, for the upwardoblique type].

Bean (1938) reported that in the Zöllner figure the haptic illusion was obtained in the same way as in the visual one. Contrary to his results, we find that the illusion obtained in the haptic mode was in the opposite direction of the visual one. Our finding could not be due to the subjects' confusion of the vertical lines with the intersected oblique lines, since the subjects reported that these lines were perceived as well-differentiated. Also, it could not be due to the possible low sensitivity of the haptic system to the line orientation, since the variability of the error scores was not different between the visual and haptic conditions. It is evident that the direction of the haptic Zöllner illusion was indeed opposite to the visual one.

\section{Delboeuf Illusion}

The results for the inner and outer circle conditions are shown in Tables 7 and 8 , respectively. For the inner circle, the illusion was obtained in the visual mode: The inner circle appeared larger than the actual $[t(15)=17.57$, $p<.0001$, one-tailed]. But no illusion was obtained in the haptic mode $[t(15)=.81$, n.s., two-tailed $]$. The difference in error scores between the haptic and visual modes was significant $[F(1,15)=18.90, p<.001]$. The left-right position did not affect the error scores $[F(1,15)=.31$, n.s. $]$.

Table 6

Mean Error Scores (in Millimeters) in the Zöllner Figures

\begin{tabular}{|c|c|c|c|c|}
\hline & \multicolumn{4}{|c|}{ Condition } \\
\hline & \multicolumn{2}{|c|}{ Downward-Oblique Type } & \multicolumn{2}{|c|}{ Upward-Oblique Type } \\
\hline & Haptic & Visual & Haptic & Visual \\
\hline $\begin{array}{l}M \\
S D\end{array}$ & $\begin{array}{l}1.0 \\
1.36\end{array}$ & $\begin{array}{r}-1.0 \\
.63\end{array}$ & $\begin{array}{r}-2.3 \\
1.17\end{array}$ & $\begin{array}{l}1.2 \\
.71\end{array}$ \\
\hline
\end{tabular}

Note-Scores are taken as the errors from the actual distance between the parallels at the top $(20 \mathrm{~mm})$. Positive values indicate the inward distortion of the parallels at the top, and negative values indicate the outward distortion.
Table 7

Mean Error Scores (in Millimeters) in the Delboeuf Figure

\begin{tabular}{lccccccc}
\hline & \multicolumn{6}{c}{ Condition } \\
\cline { 2 - 5 } & \multicolumn{3}{c}{ Haptic } & & & \multicolumn{3}{c}{ Visual } \\
\cline { 2 - 5 } \cline { 5 - 7 } & $M$ & $S D$ & Ratio & & $M$ & $S D$ & Ratio \\
\hline Left & .4 & 1.85 & 1.02 & & 2.4 & .78 & 1.12 \\
Right & .4 & 2.24 & 1.02 & & 2.6 & .77 & 1.13 \\
$M$ & .4 & 1.98 & 1.02 & & 2.5 & .56 & 1.13 \\
\hline
\end{tabular}

Note-The diameter of the inner circle was $20 \mathrm{~mm}$. Left and Right refer to the comparison stimulus on the left and on the right. Ratio is the magnitude of the illusion, that is, the ratio of the length of the comparison shaft to that of the standard shaft.

Table 8

Mean Error Scores (in Millimeters) in the Delboeuf Figure

\begin{tabular}{|c|c|c|c|c|c|c|}
\hline & \multicolumn{6}{|c|}{ Condition } \\
\hline & \multicolumn{3}{|c|}{ Haptic } & \multicolumn{3}{|c|}{ Visual } \\
\hline & $M$ & $S D$ & Ratio & $M$ & $S D$ & Ratio \\
\hline Left & -1.4 & 1.22 & .95 & -.8 & .49 & .97 \\
\hline Right & -8 & 1.72 & .97 & -.7 & .66 & .98 \\
\hline$M$ & -1.1 & 1.28 & .96 & -.8 & .46 & .97 \\
\hline
\end{tabular}

Note-The diameter of the outer circle was $30 \mathrm{~mm}$. Left and Right refer to the comparison stimulus on the left and on the right. Ratio is the magnitude of the illusion, that is, the ratio of the length of the comparison shaft to that of the standard shaft.

For the outer circle, the illusion was obtained in both the visual and haptic modes $[t(15)=6.70, p<.0005$, one-tailed, and $t(15)=3.50, p<.01$, two-tailed, respectively]. That is, the outer circle was perceived as smaller than the actual size. There was no difference in error scores between the two sensory modes $[F(1,15)=1.32$, n.s.]. The left-right position did not affect the illusion $[F(1,15)=2.48$, n.s. $]$.

The result that no size illusion of the inner circle $\alpha$ curred coincides with Hatwell's (1960), but not with Fry's (1975), results. For the outer circle, a size illusion similar to the visual one was obtained. The visual Delboeuf illusion is generally thought to be an illusion of size contrast, but an illusion of size assimilation or little illusion has been reported to occur in some modified versions of the Delboeuf figures (e.g., modified ratios of the diameter of the double concentric circles; Ogasawara, 1952). Thus, we can suppose that the manner in which the size contrast and assimilation operate is different between the haptic and visual modes.

\section{GENERAL DISCUSSION}

Although Révész (1934) reported that haptic illusions could be induced in almost all of the geometrical visual illusion figures, the results obtained in the present study suggest that this is not the case: Some geometrical illusions occurred in the haptic mode, whereas others did not. In the Müller-Lyer, vertical-horizontal, and Ponzo figures, we obtained haptic illusions equivalent to the visual ones. In the Oppel-Kundt figure, a haptic illusion similar to the visual one was obtained. In the Poggendorff fig- 
ure, no haptic illusion was obtained. In the Zöllner figure, a haptic illusion was obtained, but its direction of illusion was opposite to the visual one. Also, in the Delboeuf figure, a size illusion was obtained for the outer circle but not for the inner circle. In general, these results suggest that in the dimension of extent the haptic illusions occur in the same manner as do the visual ones, but not always in the dimensions of shape and direction.

Some researchers have suggested that haptic illusions are not purely haptic but mediated by visualization. In defense of Gregory's hypothesis for the geometrical illusions, Frisby and Davies (1971) claimed that the haptic Müller-Lyer illusion was mediated by visualization, since the magnitude of the Müller-Lyer illusion correlated between the haptic and visual modes. Appelle and Gravetter (1985) also suggested that the haptic oblique effect was mediated by visual imagery, since the effect was more susceptible to the visual experience than to the haptic one. Thus, the geometrical haptic illusions obtained in the present study might also be mediated by visualization. However, the absence of a haptic illusion in some figures, as well as the discrepancy between the haptic and visual illusions in some figures, suggest that this possibility of visualization can be ruled out. If haptic illusions had been mediated by visualization, they would have occurred in all of the figures in a manner similar to that of the visual illusions. But this was not the case. The haptic illusions obtained in the present study seemed to be essentially haptic, not necessarily mediated by visualization. This idea is supported by the findings that the haptic Müller-Lyer illusion was obtained in the congenitally blind, who had not had any experience of visualization (Bean, 1938; Patterson \& Deffenbacher, 1972).

The observed equivalence between the visual and haptic modes in some illusions in the present study, however, does not always mean that the same or similar processes were at work in these two modalities. As shown by Cheng (1968), Day and Wong (1971), and Wong (1977), the haptic vertical-horizontal illusion depends on the difference between radial and tangential movements of hand and arm. The speed of the hand and arm movement differs according to the direction relative to the body, and this determines the perceived length of the lines. Thus, the observed equivalences between the visual and haptic illusions might be superficial ones. That is, even if the magnitude of the illusion were the same in the two modes, the determinants of the illusion might differ between the two modes. This might be true in the other illusions obtained in both modes. Together with Wenderoth and Alais's (1990) finding of no haptic Poggendorff illusion, our results that in the haptic mode little illusion or an illusion directionally opposite to the visual one was observed in the Poggendorff, Zöllner, and Delboeuf figures can be understood as showing that different strategies are taken between the two modes. Klatzky and Lederman (1987) also showed that these two modalities take different encoding strategies. However, it is also possible that they take similar strategies. This might be the case in the Müller-Lyer illusion, since the magnitudes of the visual and haptic illusions correlate with each other (Frisby \& Davies, 1971) and the intermodal negative transfer of the illusion occurs between these two modes (Over, 1966; Rudel \& Teuber, 1963).

As for the Delboeuf and Zöllner illusions, in which a discrepancy between the visual and haptic modes was found, we can say that the manner in which contrast and assimilation operate differs between the two modalities. In the Zöllner figures used in the present study, it can be said that vertical lines and intersecting oblique lines influenced each other in an assimilative way in the haptic mode but in a contrastive way in the visual mode. In the visual mode, an illusion directionally opposite to the normal Zöllner illusion can be obtained when the intersection angle in the Zöllner figure is small (Tyler \& Nakayama, 1984). Our results are comparable to this directionally opposite Zöllner illusion. Thus, the effects of contrast and assimilation of line orientation might differ depending on the intersection angle of lines in each sensory mode. In the Delboeuf figure used in the present study, the haptic size illusion occurred for the outer circles, but not for the inner circles. Although the visual Delboeuf illusion is generally thought to be an illusion of size contrast, Ogasawara (1952) reported that an illusion of size assimilation or no illusion can be induced in some modified versipns of the Delboeuf figures. Thus, also in the Delboeuf figure, which effect, size contrast or assimilation, can be induced depends on the figural conditions, and the appropriate conditions for each effect might differ between two modes.

In the Poggendorff figures, no haptic illusion was obtained. In their article reporting the absence of the haptic Poggendorff illusion, Wenderoth and Alais (1990) suggested that it could not be explained with regard to the manner of exploration (i.e., the simultaneous way in vision vs. the sequential way in haptics), because the visual Poggendorff illusion could be induced by the successive presentation of the oblique lines (Wenderoth \& Johnson, 1983). Wenderoth and Alais instead suggested the lack of visualization as one of the possible reasons why they could not obtain the haptic illusion. That is, if the subjects can profitably use visualization by practice, the haptic Poggendorff illusion might occur. Although it is worth investigating, we at present suppose that the Poggendorff illusion does not occur in the haptic mode proper.

\section{REFERENCES}

Appelle, S., \& Gravetter, F. (1985). Effect of modality-specific experience on visual and haptic judgment of orientation. Perception, 14, $763-773$.

BEAN, C. H. (1938). The blind have "optical illusions." Journal of Experimental Psychology, 22, 283-289.

BÉKÉSY, G. VON (1967). Sensory inhibition. Princeton, NJ: Princeton University Press.

Cheng, M. F. H. (1968). Tactile-kinesthetic perception of length. American Journal of Psychology, 81, 74-82.

Coren, S., Girgus, J. S., Erlichman, H., \& Hakstian, A. R. (1976). An empirical taxonomy of visual illusions. Perception \& Psychophysics, 20, 129-137. 
CralG, F. E. (1931). Variations in the illusion of filled and unfilled tactual space. American Journal of Psychology, 43, 112-114.

DAY, R. H., WONG, T. S. (1971). Radial and tangential movement directions as determinants of the haptic illusion in an L figure. Journal of Experimental Psychology, 87, 19-22.

Frisby, J. P., DAvies, I. R. L. (1971). Is the haptic Müller-Lyer a visual phenomenon? Nature, 231, 463-465.

Fry, C. L. (1975). Tactual illusions. Perceptual \& Motor Skills, 40. 955-960.

GREGORY, R. L. (1963). Distortion of visual space as inappropriate constancy scaling. Nature, 199, 678-680.

HATWELL, Y. (1960). Étude de quelque illusions géométriques tactiles chez les aveugles. L'Année Psychologique, 60, 11-27.

Klatzky, R. L., Lederman, S. J. (1987). There's more to touch than meets the eye: The salience of object attributes for haptics with and without vision. Joumal of Experimental Psychology: General, 116, 356-369.

Lucca, A., Dellantonio, A., Riggio, L. (1986). Some observations on the Poggendorff and Müller-Lyer tactual illusions. Perception \& Psychophysics, 39, 374-380.

Ogasamara, J. (1952). Displacement effect of concentric circles. Japanese Journal of Psychology, 22, 224-234. (In Japanese with English summary)

OVER, R. (1966). A comparison of haptic and visual judgments of some illusions. American Journal of Psychology, 79, 590-595.

OVER, R. (1967). Haptic illusions and inappropriate constancy scaling. Nature, 214, 629.

PARRISH, C. S. (1895). The cutaneous estimation of open and filled space. American Joumal of Psychology, 6, 514-520.
Pasnak, R. \& Ahr, P. (1970). Tactual Poggendorff illusion in blind and blindfolded subjects. Perceptual \& Motor Skills, 35, 151-154.

Patterson, J., \&effenbacher, K. (1972). Haptic perception of the Müller-Lyer illusion by the blind. Perceptual \& Motor Skills, 35 , 819-824

RÉvész, G. (1934). System der optischen und haptischen Raumtäuschungen. Zeitschrift fur Psychologie, 131, 292-375.

Rudel, R. G., Teuber, H. L. (1963). Decrement of visual and haptic Müller-Lyer illusion on repeated trials: A study of crossmodal transfer. Quarterly Journal of Experimental Psychology, 15, 125-131.

Tsai, S. (1967). Müller-Lyer illusion by the blind. Perceptual \& Motor Skills, 25, 641-644.

TYLER, C. W.. NAKAyAma, K. (1984). Size interactions in the perception of orientation. In L. Spillman \& B. R. Wooten (Eds.), Sensory experience, adaptation, and perception (pp. 529-546). Hillsdale. NJ: Erlbaum.

WENDEROTH, P., ALAIS, D. (1990). Lack of evidence for a tactual Poggendorff illusion. Perception \& Psychophysics, 48, 234-242.

WENDEROTh, P., Johnson, M. (1983). Relationships between the kinetic, alternating-line, and Poggendorff illusions: The effects of interstimulus interval, inducing parallels, and fixation. Perception \& Psychophysics, 34, 273- 279.

Wong, T. S. (1977). Dynamic properties of radial and tangential movements as determinants of the haptic horizontal-vertical illusion with an L figure. Journal of Experimental Psychology: Human Perception \& Performance, 3, 151-164.

(Manuscript received July 10, 1991; revision accepted for publication March 10, 1992 